\title{
Evaluation of Drug Resistance from Sputum Specimens of Suspected Tuberculosis Patients attending a District Hospital, Abuja
}

Abbas Abel Anzaku ( $\sim$ humbleabel2016@yahoo.com )

Institute of Human Virology

Research article

Keywords: Drug resistance, M. tuberculosis complex, antimicrobial susceptibility, Abuja, sputum, first-line drugs

Posted Date: May 7th, 2019

DOI: https://doi.org/10.21203/rs.2.9513/v1

License: (c) (i) This work is licensed under a Creative Commons Attribution 4.0 International License.

Read Full License 


\section{Abstract}

Background: This study evaluated the prevalence of drug resistant tuberculosis infection among suspected tuberculosis patients attending Asokoro District Hospital (ADH), Abuja, Nigeria. Methods: A total of $301 \mathrm{AFB}+/$ - sputum specimens from suspected TB patients were collected for the study following study approval from Asokoro District Hospital Ethic Committee. Eighty-nine (89) identified Mycobacterium tuberculosis complex were isolated after morphological and biochemical testing. Sputum specimens collected from the patients were processed, cultured on Lowenstein-Jensen (LJ) media and subjected to in-vitro antimicrobial susceptibility testing. The eighty-nine (89) identified M. tuberculosis complex isolates were challenged with Rifampicin (RIF), Isoniazid (INH), Ethambutol (EMB) and Streptomycin (SM) drugs respectively. Results: Out of the total 89 samples analyzed, 13 (14.6\%) showed resistance to the first-line drugs while $76(85.4 \%)$ were susceptible. Drug resistance was identified in 10 (11.8\%) of the new tuberculosis case (primary drug resistance cases) and $3(75 \%)$ in re-treatment cases (secondary or acquired resistance). Prevalence of mono-resistant, double resistant and triple resistant patterns were $5(5.7 \%), 6(6.7 \%)$ and $2(2.2 \%)$ respectively. Multi-drug resistant (resistant to both RIF and $\mathrm{INH}$, the two most potent anti-tuberculosis drugs, with or without resistant to other drugs) was $1.1 \%$. No isolate was resistant to all the four drugs. Conclusion: Drug resistance were observed in the cultures of M. tuberculosis complex, however no isolate showed resistance to all the four first-line drugs used. This study therefore recommends strict adherent to medication for tuberculosis patients as poor compliance or inappropriate use of drugs could induce resistance. Keywords: Drug resistance, M. tuberculosis complex, antimicrobial susceptibility, Abuja, sputum, first-line drugs.

\section{Introduction}

Nigeria is one of the leading countries in the high burden countries for TB/HIV, DR-TB, MDR-TB and XDRTB with an estimated rate of 322 per 100000 population with only $15 \%$ of the total burden of the disease in the country being reformed in 2015 (WHO, 2017) According to WHO (2017), out of the global rate of 10.4 million incident cases of TB, 3.9\% were estimated to have had rifampicin-or Drug resistant tuberculosis whereas $21 \%$ of previously treated TB cases were estimated to have had MDR/RR-TB in the same year. Nigeria is one of the leading countries in the high burden countries for TB/HIV, DR-TB, MDR-TB and XDR-TB with an estimated rate of 322 per 100000 population with only $15 \%$ of the total burden of the disease in the country being reformed in 2015 (WHO, 2017). MDR-TB is caused by strains of M. tuberculosis that is resistant to both isoniazid and rifampicin. Drug-resistant TB (DR-TB) patients require prolonged and expensive treatment using second-line medications that are less effective and more toxic (WHO, 2016). Anzaku et al (2017), reported that the control efforts and the burden of drug resistant tuberculosis (TB) is poorly comprehended in resource-limited settings.

Multi-drug resistant tuberculosis (MDR-TB) (resistant to rifampicin and isoniazid, the two most powerful first-line TB drugs) could be worse, and the emergence of more potent extensively drug-resistant (XDR) $M$. tuberculosis (defined as resistant to at least isoniazid, rifampin, any fluoroquinolone, and either aminoglycosides [amikacin, kanamycin] or streptomycin or both) in Africa and worldwide has been a 
major challenge in tuberculosis control worldwide (WHO, 2012). As part of the strategies to trash tuberculosis, the Nigerian government established the National TB and Leprosy Control Programme (NTBLCP) in 1991 and subsequently adopted the WHO-recommended Directly Observed Treatments (DOTs) strategy in 1993 to checkmate the scourge of TB (PHW, 2006). DOTs has since expanded from its previous $47 \%$ coverage areas of 1995 to the present $75 \%($ WHO, 2008) with little impact on TB spread as Nigeria stands shoulder high among the 22 high burden countries with new cases. The current cases detection rate of $20 \%$ and treatment success of $75 \%$ (WHO, 2008) has not fully meet up with the universally recommended $70 \%$ new sputum smear-positive case detection arising per year and $85 \%$ successful treatment of such cases (Dye et al., 2007). Between 1995 and 2006 the case detection rate has increased from 11 percent to 20 percent while the treatment success rate slipped from $49 \%$ in 1995 to $32 \%$ in 1996 and picked in 1997 to $73 \%$ (WHO, 2015). The treatment success rate has maintained the above $70 \%$ since then. The 2004 incidence figure of tuberculosis was 290/100,000 persons per year or 373,682 new TB cases of which 33,755 (126/100,000) were smear positive (WHO, 2016). However, the new WHO report (WHO, 2015) put the incidence case at 311/100,000 person or estimated 450,000 new cases of which $137 / 100000$ or 198,300 persons are smear positive.

Although the true level of drug resistance tuberculosis in the world today is unknown, studies have shown that it has been increasing (Espinal et al., 2011). Drug resistant surveillance commenced in the developed world in the early 1990s but the true incidence remained unclear in the developing world where there is neither no or limited access to testing for drug susceptibility (Cohn et al., 2010). An estimated $4 \%$ of the world's tuberculosis cases are resistant to at least one of the current first-line drugs (Moran, 2014). WHO reported global burden of drug resistant tuberculosis data in order to determine the global magnitude of resistance to the first-line antituberculosis drugs and by 2003, the third round of the global project (WHO, 2004). Despite strategies to curb emergence of drug resistance tuberculosis, most TB drugs are increasingly becoming resistance (WHO, 2009). This study was conducted to determine the prevalence of anti-tuberculosis drug resistance in a selected district hospital in Abuja, a section of Nigeria.

\subsection{Materials And Methods}

De-identified sputum specimens obtained from suspected TB patients attending Asokoro District Hospital (ADH), Abuja collected from the laboratory between February 2015 and July 2016 were studied in the Research Laboratory of Zankli Medical Centre in Abuja.

\subsection{Ethical clearance and informed consent form}

Approval for the study was obtained from Asokoro Disctrict Hospital Ethic Committe and sputum from patients with no reported history of prior antituberculosis treatment and those that have previously accessed therapy but not on TB treatment as at the start of this work were used. Patients were properly counselled on the importance of the study and Informed consent was obtained from all participants. Those below 18 years had their parents or guidance endorsed the consent in proxy. Both patients and the 
hospital management were also informed that the study would not interfere with the patients' management.

\subsection{Sputum sample processing}

Sputum specimens were processed according to standard mycobacteriological culture method while Mycobacterium tuberculosis complexwere identified by microscopy, morphology and biochemical tests respectively(WHO, 2015). Sputum specimens were digested and decontaminated using the modified Petroff's method (WHO, 1998). $100 \mu$ l of sediments (decontaminant) from each specimen was cultured on $\mathrm{LJ}$ media and incubated at $37^{\circ} \mathrm{C}$ for 30 days to observe for growth. The isolates were confirmed as $\mathrm{M}$. tuberculosis complex (MTBC) by cultural characteristics, nitrate reduction test, $68^{\circ} \mathrm{C}$ catalase test and Ziehl Neelsen smear to observe the serpentine cords typical of MTBC.

Drug susceptibility of the isolates to Rifampicin, Isoniazid, Ethambuthol and Streptomycin was done by Proportion method (Kehinde, 2012). The recommended critical concentrations for the drugs were $4 \mu \mathrm{g} / \mathrm{ml}$ for SM, $0.2 \mu \mathrm{g} / \mathrm{ml}$ for INH, $40 \mu \mathrm{g} / \mathrm{ml}$ for RIF and $2 \mu \mathrm{g} / \mathrm{ml}$ for EMB respectively. The drug resistant was determined by the ratio of number of colonies growing in drug-containing medium and on drug-free medium and more than $1 \%$ increase was considered to be resistant (Vareldzis et al., 1994; Canetti et al., 1969; Canetti et al., 1963).

\subsection{Primary resistance}

Primary resistance was classified as resistance to at least one of the drugs tested on the isolates from patient without prior history of anti-tuberculosis therapy while acquired resistant was classified as resistance of isolate obtained from patient that has previously being treated for tuberculosis. Multidrug resistant tuberculosis (MDR-TB) was classified as resistant to at least both the rifampicin and isoniazid.

Statistical analysis was done using OpenEpi version 2.2 software packages with variables subjected to Pearson's Chi-squared tests or Fisher exact tests when numbers were small.

\subsection{Results And Discussion}

The growth response of the sputum specimens on LJ media is summarized below. Of three hundred and one (301) AFB+ and - cultures studied, ninety-seven (97) mycobacteria isolates grew, 190 did not grow and the remaining 14 were contaminated. Of the 97 isolates, 89 were MTB complex while 8 were identified as non-tuberculous mycobacteria (NTM) (Table 1).

Table 1: Culture status of AFB+/-sputum specimens on LJ media

The result of anituberculosis of the drug resistance tuberculosis to both the first line drugs and second line drugs is presented in table 2 below. The prevalence of drug resistance in this study was $14.6 \%$ while 
$85.4 \%$ were susceptible. Primary drug resistance was $11.8 \%$ (10/85) while secondary or acquired resistance was $5 \%(3 / 4)$ respectively. The overall prevalence of MDR-TB was $1.1 \%$. Mono-resistant TB case was $5.7 \%$ with the highest occurring from streptomycin resistant $3.4 \%$ follow by ethambutol $2.3 \%$. Double resistant TB case was $6.7 \%$ with combination of rifampicin-streptomycin (1.1\%) and isoniazidstreptomycin combination (5.6\%). Triple resistant tuberculosis case prevalence was $2.2 \%$. Rifampicinisoniazid-streptomycin combination and isoniazid-ethambutol-streptomycin combination were the only two identified cases in this category. The prevalence of rifampicin-isoniazid-streptomycin resistance was $1.1 \%$ and that of isoniazid-ethambutol-streptomycin was also $1.1 \%$. From this study, double resistance had the highest prevalence rate (6.7\%) follow by mono-resistant $(5.7 \%)$ and triple resistant $(2.2 \%)$ which had the lowest rate. The figure below showed the percentage profile of the resistance prevalent.

Table 2: Prevalence of drug resistance tuberculosis= $(14.6 \%)$

Table 3. Drug Resistant profile of M. tuberculosis complex isolates from Asokoro District Hospital, Abuja.

Figure 1 below showed total tested isolates, resistance isolates and susceptible isolates to antituberculosis agents.

\subsection{Discussion}

The prevalence of drug resistance in this study was $14.6 \%$. Levels of primary and secondary drug resistance are $11.8 \%$ and $75 \%$ respectively among suspected tuberculosis patients attending the district hospital in Abuja. The level of acquired resistant in this study is significantly higher than the primary resistant $(p=0.016)$. Meanwhile, the primary and acquired drug resistance fall below the acceptable limit for country with well implemented national tuberculosis control programme. Level of acquired drug resistance is between $50 \%-80 \%$ where the national tuberculosis control programme is poorly implemented (Crofton et al., 2012). As acquired drug resistance determines the occurrence of primary resistance, the latter is expected to be lower than the former (Chaulet et al., 2015). Okodua et al. (2012), asserted that primary drug resistant tuberculosis is $5 \%$ or less and acquired drug resistant to decrease to between $10 \%-20 \%$ where DOTS program is well implemented. This study shows $1.1 \%$ rate of multi-drug resistant tuberculosis (MDR-TB) which was in contrast to Lawson et al., (2011) that reported 8\% cases of MDRTB. This could be as a result of three (3) cities covered for their study and a larger sample size as compared to one district hospital for this present study. The prevalence of MDR-TB among the re-treatment cases in this study was $25 \%$. This was about similar to nationwide survey report of $25.6 \%$ in China (WHO, 2010) and contrary to another study from Jos (a North central city in Nigeria) that reported $18 \%$ of retreatment cases (Ani et al., 2009). This could be because most patients accessing the DOTS may not have enough information on importance of drug adherence as it is often noticed that they stop their regimen without completing it once they get relief (PHW, 2006). MDR-TB is a public health concern as it accounts for TB treatment failure worldwide (WHO, 2014). Identifying patients with MDR-TB earlier enables rapid treatment which increases their chances of survival and minimizes risk of transmission (Breathnach et al., 2008). However, treatment of MDR-TB is complicated because it requires the use of second-line drugs 
for $\geq 24$ months which are more costly, toxic, and less effective than the first-line drugs used for regular tuberculosis therapy (Shah. et al., 2007). The results of monoresistant pattern showed $5.7 \%$ prevalence. Resistant to specific drug indicates that Streptomycin and ethambutol are observed with prevalence of $3.4 \%$ and $2.3 \%$ respectively. The range of resistant to these two drugs is also in consistent with the world report resistant range of 0-4.9\% for Streptomycin and 0-4.2\% for ethambutol respectively found in countries with cases of monoresistant tuberculosis challenge (WHO, 2004).

Double resistant pattern in this study show resistant MTB isolates to: (1) 5.6\% prevalence in Isoniazid and streptomycin combination. (2) $1.1 \%$ prevalence in Rifampicin and streptomycin combination. The Isoniazid and streptomycin combination prevalence could be because they are among the first few antiTB in existence since middle of nineteen century, hence resistant to these two old drugs may have developed over the years (Bello, 2014). Of concern is the isolate resistant to both rifampicin and streptomycin (1.1\%). Although MDR-TB is resistant to at least rifampin and isoniazid, the two most powerful and commonly used anti-TB drugs WHO (2014), resistant to RIF alone can be a useful surrogate marker for MDR-TB (Sam et al., 2006; WHO, 2004a; Somoskovi et al., 2001). MDR-TB threatens the effectiveness of chemotherapy for both treatment and control of TB and require the use of second-line drugs that are more expensive, less effective than the first-line drugs, toxic and require longer administration periods (18-24 months) (Prammananan et al., 2005; Moran, 2004). Mortality among cases of MDR-TB is high and this is worrisome because of its high treatment failure rate. Idigbe et al. (2002), reported that $56 \%$ of the strains recovered from 96 patients not responding to anti-TB treatment were resistant to one or more of the drugs used, with $38 \%$ being resistant to isoniazid, although only $2 \%$ were resistant to rifampicin at that time. Triple resistant pattern of the MTB in this study show: (1) $1.1 \%$ prevalence of Rifampicin, isoniazid and streptomycin combination. (2) $1.1 \%$ prevalence of Isoniazid, ethambutol and streptomycin combination. Isolate showing resistant to rifampicin and isoniazid with or without other first line drug is classified to be a MDR-TB (Sam et al., 2006; WHO, 2004a; Somoskovi et al., 2001), often identified with high mortality among the victims and threat to TB treatment and control. The second triple resistant case may be because these drugs were among the first anti-tuberculosis drugs (especially for isoniazid and streptomycin) (Johnson et al., 2007; Crofton and Mitchison, 1948), and it being abused or inappropriately used over the years. However, EMB resistant in this triple resistant may not be categorical because phenotypic determination of true resistant to EMB is still unclear (Johnson et al., 2007; Johnson et al., 2006).

The overall drug resistant TB rate of $14.6 \%$ found in this study is attributable to poor compliance with the DOTS guidelines common in low-income countries (Jones-Lopez and Ellnerr, 2005), irrational use of drugs as common in countries that sell antimicrobial drugs without prescription (i.e., over-the-counter) (Stratchounski et al., 2013; Okumura et al., 2012), lack of the DOTS in parts of the remote areas in the country (PHW, 2006), proliferation of unregistered drug distributors in resource poor settings like Nigeria, inadequate awareness about tuberculosis (its mode of infection and treatment), poor socio-economic condition, HIV/AIDS as well as illiteracy. 


\subsection{Conclusion And Recommendation}

Drug resistance were observed in the identified cultures of $M$. tuberculosis complex, however no isolate showed resistance to all the four first-line drugs used. This study therefore recommends strict adherent to medication for tuberculosis patients as poor compliance or inappropriate use of drugs could induce resistance.

Strengthening Laboratory capacity that include drug resistance surveillance in Nigeria should be encouraged. This could be done through the provision of faster, automated culture and DST facilities or automated polymerase chain reaction (PCR) technologies that are emerging elsewhere. Future studies should investigate if extensively drug resistant TB (XDR-TB) strains are also affecting newly diagnosed patients with TB in Nigeria and if strains are still sensitive to fluroquinolones and other alternative agents.

\section{Declarations}

\section{Competing interest}

All authors have declared that, no conflict of interest exist in this manuscript.

\section{Ethical clearance:}

The ethical clearance for this study was obtained from the Ethic Committee of Asokoro District Hospital, Abuja, Nigeria. Participants for this study were allowed to voluntarily participate after obtaining informed consent forms.

\section{Acknowledgement:}

We will like to thank the Asokoro District Hospital, Abuja for study approval and Zankli Medical Centre for supporting the research.

\section{References}

Ani AE, Daylop YB, Agbaji O \& Idoko J (2009). Drug susceptibility test of Mycobacterium tuberculosis by nitrate reductase assay. Journal of Infection in Developing Countries 3: 16- 19.

Bello LA, Shittu MO, Shittu BT, Oluremi AS, Akinnuroju ON, Adekola SA. Rifampicin- monoresistant Mycobacterium tuberculosis among the patients visiting chest clinic, state specialist hospital, Akure, Nigeria. Int J Res Med Sci. 2014; 2:1134-1137.

Breathnach, A.S., de Ruiter, A., Holdsworth, G.M., Bateman, N.T., O'Sullivan, D.G and Rees, P.J. (2008). An outbreak of multidrug resistant tuberculosis in a London teaching hospital. J. Hosp. Infect. 39:111-7. 
Chaulet, P., Boulahbal, F., and Grosset, J. (2015). Surveillance of drug resistance for tuberculosis control: why and how? Tuberc. Lung Dis. 76 (6): 487-492.

Crofton, J., Chauletxs nx, P., and Maher. D (2012). Guidelines for the management of drug- resistant tuberculosis. WHO/TB/96.210 (Rev.1). World Health Organization, Geneva, Switzerland.

Canetti, G., Fox, W., Khomenko, A., Mahler, H.T., Menon, N.K., Mitchison, D.A., et al., (1969). Advances in techniques of testing mycobacterial drug sensitivity, and the use of sensitivity tests in tuberculosis control programmes. Bull World Health Organ 41: 21-43.

Canetti, G., Froman, S., Grosset, J et al., (1963). Mycobacteria: laboratory methods for testing drug sensitivity and resistance. Bull World Health Organ 29: 565-78.

Chaulet, P., Boulahbal, F., and Grosset, J. (1995). Surveillance of drug resistance for tuberculosis control: why and how? Tuberc. Lung Dis. 76 (6): 487-492.

Cohn, D.L., Bustreo, F., and M.C. Raviglione (1997). Drug-resistant tuberculosis: review of worldwide situation and the WHO/IUATLD Global Surveillance Project. Clin. Infect. Dis. 24 Suppl 1, S121-S130.

Crofton, J., Chauletxs nx, P., and Maher. D (1997). Guidelines for the management of drug- resistant tuberculosis. WHO/TB/96.210 (Rev.1). World Health Organization, Geneva, Switzerland.

Crofton, J. (1994). Multidrug resistance: danger for the Third World. In Porter JDH, McAdam, KDNJ. Editors: "Tuberculosis back to the future", Chichester, John Wiley \& Sons Ltd., 231-233.

Crofton, J and Mitchison, D.A. (1948). Streptomycin resistance in pulmonary tuberculosis. Brit. Med. J. 2:1009-1015.

Barnes, P.F., and Barrows, S.A (1993). Tuberculosis in the 1990's. Ann Intern Med 119: 400-10.

Bloch, A.B., Cauthen, G.M., and Onorato, I.M. (1994). Nationwide survey of drug-resistant tuberculosis in the United States. J. Amer. Med. Assoc. 271:665-671.

Breathnach, A.S., de Ruiter, A., Holdsworth, G.M., Bateman, N.T., O'Sullivan, D.G and Rees, P.J. (1998). An outbreak of multidrug resistant tuberculosis in a London teaching hospital. J. Hosp. Infect. 39:111-7.

Braun, M.M., Kilburn, J.O., Smithwick, R.W, et al., (1992). HIV infection and primary resistance to antituberculosis drugs in Abidjan, Cote d'Ivoire. AIDS 6: 1327-1330.

Dye, C., Hosseini, M., and C. Watt (2007). Did we reach the 2005 target hjhijio for tuberculosis control? Bull World Health Organ 85: 364-9.

Donald, E., and A. V. Rie (2006).XDR tuberculosis: an indicator of public health Negligence. Lancet 368:1554-1556. 
Drobniewski, F., Eltringham, I., Graham, C., Magee, J.G., Smith, E.G and Watt, B. (2002). A national study of clinical and laboratory factors affecting the survival of patients with multiple drug resistant tuberculosis in the UK. Thorax 57: 810-6.

Dye, C., Hosseini, M., and C. Watt (2007). Did we reach the 2005 target hjhijio for tuberculosis control? Bull World Health Organ 85: 364-9.

Espinal, M.A., Adalbert, P.H., Laszlo, A.L., et al., (2003). Global trends in resistance to antituberculosis drugs. N Engl J Med 344: 1294-303.

Espinal, M.A., Laserson,K., Camacho, M., Fusheng, Z., Kim, S.J., Tlali, R.E., Smith, I., Suarez, P., Atunes, M.L George, A.G., Martin-Casabona, N., Simelane, P., Weyer, K., Binkin, N., Raviglione, M,C (2001). Determinants of drug-resistant tuberculosis: analysis of 11 countries. Int J Tuberc Lung Dis 5(10): 887-893.

Raviglione, M,C (2001). Determinants of drug-resistant tuberculosis: analysis of 11 countries. Int J Tuberc Lung Dis 5(10): 887-893.

Gandhi, N. R., A. Moll, A. W. Sturm, R. Pawinski, T. Govender, U. Lalloo, K. Zeller, J. Andrews, and G. Friedland (2006). Extensively drug-resistant tuberculosis as a cause of death in patients co-infected with tuberculosis and HIV in a rural area of South Africa. Lancet 368:1575-1580.

Grange, J.M., and Zuma, A (2002). The global emergency of tuberculosis: What is the cause? J. Royal Soc Health 122: 78-81.

Gupta, R., Kim, J.Y., Espinal, M.A., Caudron, J.M., Farmer, P.E., and Raviglione, M.C. (2001). Responding to market failures in tuberculosis: a model to increase access to drugs and treatment. Science 293:1049-51.

Horsburgh, C.R. (2000). The global problem of multidrug-resistant tuberculosis: The genie is out of the bottle. J. Amer. Med. Assoc. 283:2575-2576.

Idigbe EO, Duque JP, John EK, Annam O. Resistance to antituberculosis drugs in treated patients in Lagos, Nigeria. J Trop Med Hyg 1992;95:186-91.

Johnson, R., Streicher, E.M., Louw, G.E., Warren, R.M., van Helden, P.D., and Victor.T.C (2007). Drug resistance in Mycobacterium tuberculosis. Mol Biol 8: 97-112.

Jordaan R, A.M., Pretorius, L., Engelke, E., van der, S.G.,Kewley, C., Bosman, M., van Helden, P.D., Warren, R., and Victor, T.C (2006). Ethambutol resistant testing by mutation detection. Int. J. Johnson Tuberc. Lung Dis 10: 68-73.

Kehinde AO, Adetoye AE. Diagnosis of pulmonary tuberculosis using genotype MTBDRPLUS assay in three local government primary health centres of Osun State, Nigeria- a pilot study. J Med Microb Diagn. 2012; S3:001. 
Kenyon, T.A., Mwasekaga, M.J., Huebner, R., Rumisha, D., Binkin, N., and E. Maganu (1999). Low levels of drug resistance amidst rapidly increasing tuberculosis and human immunodeficiency virus co-epidemics in Botswana. Int J Tuberc Lung Dis 3(1): 4-11

Kochi, A., Vareldzis, B., Styblo, K. (1994). Multidrug resistant tuberculosis and its control. Res. Microbiol., 144: 104-110.

Lawson L, Yassin M A, Abdurrahman ST. et al., (2011). Resistance to first-line tuberculosis drugs in three cities of Nigeria. Tropical Medicine and International Health 16 (8) pp 974-980

Moran, M. (2014). Running out of breath? TB care in the 21st century. MSF. pp. 1-32.

Kent, P. T., and G. P. Kubica (1985).Public health mycobacteriology: a guide for the level III laboratory. Centers for Disease Control, Atlanta, GA.

Moran, M. (2004). Running out of breath? TB care in the 21st century. MSF. pp. 1-32.

Nathanson, E., Lambregts-van, W.C., Rich, M.L., Gupta, R., Bayona, J., Blondal, K., et al., (2006). Multi-drug resistant tuberculosis management in resource-limited settings. Emerg Infect Dis 12: 1389-97.

Okodua M, Ihongbe J, Esumeh F. Pulmonary tuberculosis and resistance pattern to first line antituberculosis drugs in a city of western Nigeria. Int J Basic Appl Innov Res. 2012; 1: 48-56.

Oluwaseun E, Akinniyi AP, Afolabi O. Primary multi-drug resistant tuberculosis among HIV seropositive and seronegative patients in Abeokuta, southwestern Nigeria. Am J Res Comm. 2013; 1:224-237.

Prammananan, T., Arjatankool, W., Chaiprasert, A., Tingtoy, N., Leechawengwong, M., Aswapokee, N., Leelaramasae, A., and Dhiraputra, C (2005). Second-line susceptibilities of Thai multidrug-resistant Mycobacterium tuberculosis isolates. Int/ J Tuberc Lung Dis. 9: 216-9.

WHO (2010). Multidrug and Extensively Drug-Resistant TB (M/XDR-TB). 2010 Global Report on Surveillance and Response. WHO, Geneva, Switzerland.

World Health Organisation. Global Tuberculosis Control: WHO report 2016. Geneva, Switzerland: World Health Organisation, 2016.

\section{Tables}

Table 1: Culture status of AFB+/- sputum specimens on LJ media 


\begin{tabular}{lll}
\hline Status & Isolates & Percentage occurrence \\
\hline MTB complex & 89 & 29.6 \\
NMTB & 8 & 2.7 \\
Contaminated & 14 & 4.7 \\
No growth & 190 & 63.1 \\
Total & 301 & $100 \%$ \\
\hline
\end{tabular}

Key: MTB=Mycobacterium tuberculosis; NMTB= Non Mycobacterium tuberculosis

Table 2: Prevalence of drug resistance tuberculosis= $\mathbf{( 1 4 . 6 \% )}$

\begin{tabular}{|c|c|c|c|c|c|}
\hline \multirow[t]{2}{*}{ Anti-TB agent } & \multicolumn{3}{|c|}{ Type of resistance } & & \multirow[b]{2}{*}{ Percentage occurrence } \\
\hline & MDR-TB & $\begin{array}{l}\text { Mono } \\
\text { resistance }\end{array}$ & Double resistance & $\begin{array}{l}\text { Triple } \\
\text { resistance }\end{array}$ & \\
\hline Ethambutol & & $2.3 \%$ & & & $5.7 \%$ \\
\hline Streptomycin & & $3.4 \%$ & & & \\
\hline RIF-SM & & & $1.1 \%$ & & $6.7 \%$ \\
\hline INH-SM & & & $5.6 \%$ & & \\
\hline RIF-INH-SM & & & & $1.1 \%$ & $2.2 \%$ \\
\hline INH-EMB-SM & & & & $1.1 \%$ & \\
\hline Total & $1.1 \%$ & $5.7 \%$ & $6.7 \%$ & $2.2 \%$ & $14.6 \%$ \\
\hline
\end{tabular}

Keys: $\mathrm{SM}=$ Streptomycin; INH= Isoniazid; EMB=Ethambutol; RIF = Rifampicin

Table 3. Drug Resistant profile of M. tuberculosis complex isolates from Asokoro District Hospital, Abuja.

AFB+

Resistant Pattem

$(\mathrm{N}=89$ )

(\%)

OR

$95 \% \mathrm{Cl}$

P-value 


$\begin{array}{lccccr}\text { Any Drug (N) * } & 10 & 11.8(10 / 85) & 0.8 & 0.3-1.9 & 0.591 \\ \text { Any Drug (RE) * } & 3 & 75(3 / 4) & 16.7 & 1.7-466.4 & 0.016 \\ \text { Any Drug (N+RE) * } & 13 & 14.6 & & \text { Reference } \\ & & & & & \\ \text { MDR-TB (N) } & 0 & 0.0 & 1.0 & 0.0-41.3 & 0.977 \\ \text { MDR-TB (RE) } & 1 & 25(1 / 4) & 29.3 & 1.5-589.8 & 0.001 \\ \text { MDR-TB (N+RE) } & 1 & 1.1 & & \text { Reference }\end{array}$

\section{Monoresistant $\perp$}

Rifampicin

Isoniazid

Ethambutol

Streptomycin

Double resistant $\perp$

Rifampicin + Streptomycin

Isoniazid + Streptomycin

\section{Triple resistant $\Perp$}

Rifampicin + Isoniazid + Streptomycin

Isoniazid + Ethambutol +Streptomycin $0^{\square} \quad 0.0$

$0^{\square} \quad 0.0$

2

2.3

$3 \quad 3.4$

1.5

0.5

0.5

$0.0-6.6$

1.000

$0.0-6.6$

1.000

Reference

$0.2-13.0$

0.684
Reference

$0.7-125.9$

0.120

$$
0.7-125.9
$$

0.120

$5 \quad 5.6$

5.2

0.0-39.4 $\quad 1.000$

\section{Monoresistant $\perp$}

Double resistant $\perp$

Triple resistant $\Perp$
$5 \quad 5.7$

6
6.7

$2 \quad 2.2$
1.0

1.1
Reference

Reference

$0.3-4.5$

0.768

1.2

0.4

$0.1-2.0$

0.281 
Any Rifampicin resistance*

Any Isoniazid resistance*

Any Ethambutol resistance*

Any Streptomycin resistance*
$2 \quad 2.2$

$\begin{array}{ll}7 & 7.9\end{array}$

3

3.4

11

12.4

0.3

$0.0-1.3$

0.101

Reference

0.4

$0.1-1.6$

0.215

1.6

$0.6-4.7$

0.335

*=Resistance to drug in question, either alone or in combination with resistance to others; $N=N e w$ cases; RE=Re-treatment cases;

$\Perp=$ Resistance to the specified drug; MDR-TB= Multidrug resistant tuberculosis; OR=odds ratio; $\mathrm{Cl}=$ confidence interval.

$\bigotimes=$ calculated by adding 0.5 to numerator and denominator (reference).

\section{Figures}




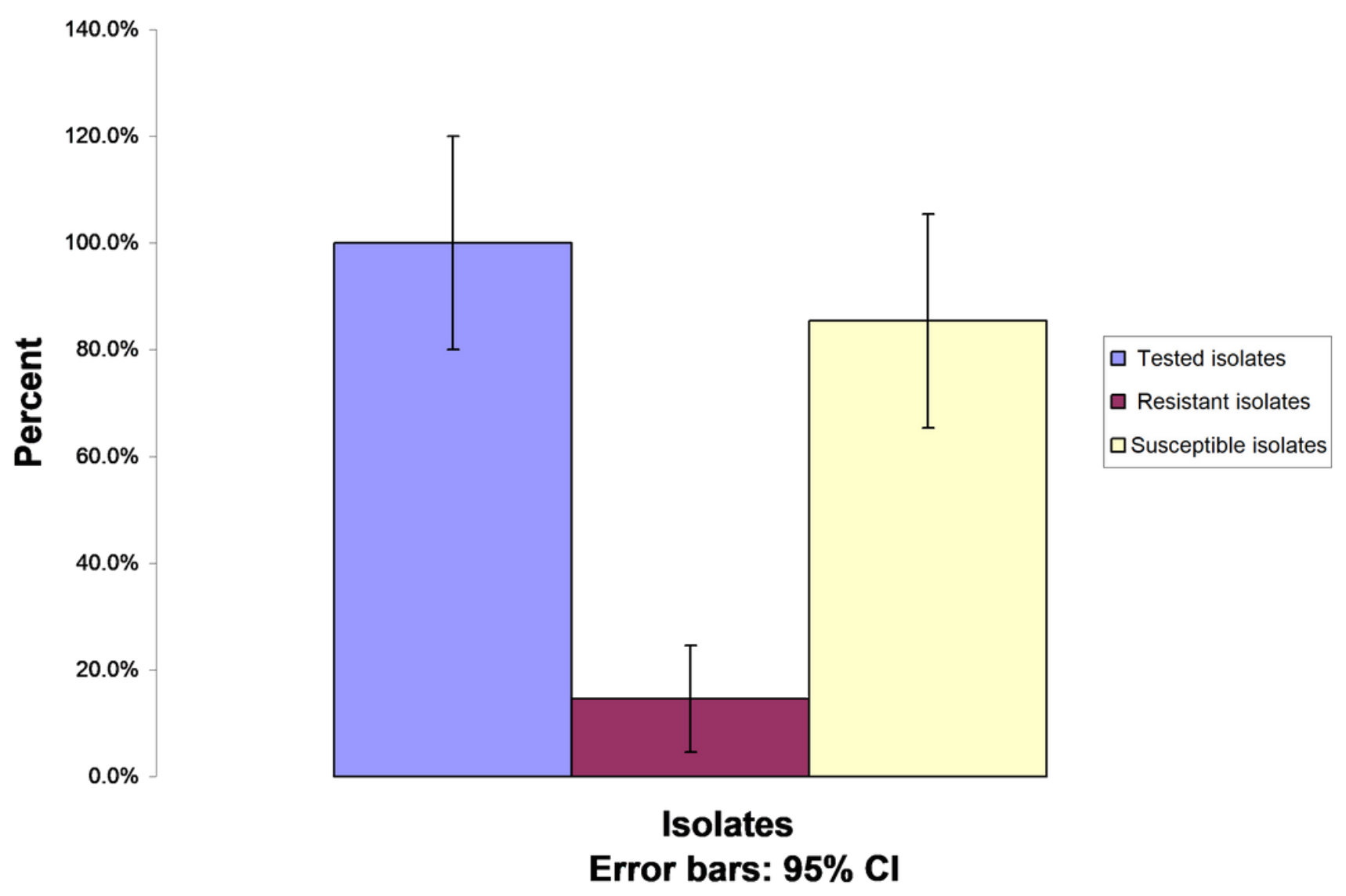

Figure 1: Isolates' response to the first-line drugs

Figure 1

Showed total tested isolates, resistance isolates and susceptible isolates to antituberculosis agents. 